\title{
Some old and new results about rigidity of critical metrics
}

\author{
GiLLES CARRON
}

\begin{abstract}
We present a new proof of a recent $\epsilon$-regularity of G. Tian and J.Viaclovsky. Our idea also provides a new proof of a classical result of M. Anderson about volume rigidity of Einstein manifolds. Eventually, we also obtain new rigidity results for critical metrics.
\end{abstract}

Mathematics Subject Classification (2010): 53C20 (primary); 58E11 (secondary).

\section{Introduction}

In this paper, we obtain some new $\epsilon$-regularity and rigidity results for critical metrics and our arguments will also give new proofs of classical $\epsilon$-regularity results.

The class of critical metrics has been introduced and studied by G. Tian and $\mathrm{J}$. Viaclovsky ([35]): A Riemannian metric $g$ is said to be critical if its Ricci curvature tensor satisfies a Bochner type formula:

$$
\nabla^{*} \nabla \operatorname{Ricci}_{g}+\mathcal{R}\left(\text { Ricci }_{g}\right)=0
$$

where $\nabla$ is the Levi-Civita connection and $\nabla^{*}$ is its differential adjoint and $\mathcal{R}$ is a linear action of the Riemann curvature tensor on the space of symmetric 2 tensors, in particular there is a constant $\Upsilon$ (that only depends on this action) such that:

$$
\forall h \in \odot^{2} T_{x}^{*} M, \quad|\mathcal{R}(h)| \leq \Upsilon|\mathrm{Rm}||h| .
$$

Examples of critical metrics are Einstein metrics, Kähler metrics with constant scalar curvature, locally conformally flat metrics with constant scalar curvature and in dimension 4, Bach flat metrics with constant scalar curvature. Our main new result is the following $\epsilon$-rigidity result:

Theorem A. There is a constant $\epsilon>0$ that only depends on the dimension $n$ and on the constant $\Upsilon$ appearing in the estimate (1.1) such that, if $\left(M^{n}, g\right)$ is a complete 
Riemannian manifold whose metric is critical and such that its Riemann curvature tensor satisfies for some fixed point $o \in M$ :

$$
|\operatorname{Rm}(y)| \leq \frac{\epsilon^{2}}{d(o, y)^{2}}
$$

then the metric $g$ is flat: $\mathrm{Rm}=0$.

Our result generalizes a recent result of V. Minerbe ([24]) who proved a similar result for Ricci flat metrics with controlled volume growth:

Theorem 1.1. Assume that $\left(M^{n}, g\right)$ is complete Ricci flat Riemannian manifold

$$
\operatorname{Ricci}_{g}=0
$$

such that for some fixed point $o \in M$, some $v>1$ and some positive constant $C>0$ :

$$
\forall R>r>0, \quad \frac{\operatorname{vol} B(o, R)}{\operatorname{vol} B(o, r)} \geq C\left(\frac{R}{r}\right)^{v}
$$

then there is a constant $\epsilon>0$ that only depends on $n, v, C$ such that if

$$
|\operatorname{Rm}(y)| \leq \frac{\epsilon^{2}}{d(o, y)^{2}}
$$

then the metric $g$ is flat: $\mathrm{Rm}=0$.

The first step in the proof of Theorem 1.1 was to establish an $L^{1}$ Hardy inequality:

$$
\forall f \in C_{0}^{\infty}(M), \mu(n, v, C) \int_{M} \frac{|f(x)|}{d(x, o)} d \operatorname{vol}_{g}(x) \leq \int_{M}|d f(x)| d \operatorname{vol}_{g}(x) .
$$

And the final step was to use the Bochner type equation

$$
\nabla^{*} \nabla \mathrm{Rm}+\mathcal{R}(\mathrm{Rm})=0
$$

satisfied by the Riemann curvature tensor of a Ricci flat metric.

There are many other $\epsilon$-rigidity results that rely on a priori functional inequalities (such as a Sobolev inequality or as the above Hardy inequality) and integral bounds on the curvature ( $c f$. for instance $[5,13,20,21,25,27,29,30,32,33]$, [35, Theorem 7.1], [38]). Such results have been shown recently for critical metrics by G. Tian and J. Viaclovsky in dimension 4 and by X-X. Chen and B. Weber in higher dimension $([12,35])$ :

Theorem 1.2. There are positive constants $\epsilon>0$ and $C>0$ that depend only on the dimension $n$ and on the constant $\Upsilon$ appearing in the estimate (1.1) such that when $\left(M^{n}, g\right)$ is a complete Riemannian manifold whose metric is critical and such 
that for some $x \in M$ and for some $r>0$, the geodesic ball $B(x, r)$ satisfies the Sobolev inequality:

$$
\forall f \in C_{0}^{\infty}(B(x, r)), \quad\left(\int_{B(x, r)}|f(y)|^{\frac{2 n}{n-2}} d \operatorname{vol}(y)\right)^{1-\frac{2}{n}} \leq A \int_{B(x, r)}|d f(y)|^{2} d \operatorname{vol}(y)
$$

and the following integral bound on the curvature tensor:

$$
A^{\frac{n}{2}} \int_{B(x, r)}|\operatorname{Rm}(y)|^{\frac{n}{2}} d \operatorname{vol}_{g}(y)<\epsilon
$$

then

$$
\sup _{B\left(x, \frac{1}{2} r\right)}|\mathrm{Rm}| \leq A \frac{C}{r^{2}}\left(\int_{B(x, r)}|\operatorname{Rm}|^{\frac{n}{2}}(y) d \operatorname{vol}_{g}(y)\right)^{\frac{2}{n}} .
$$

When the radius $r$ tends to $\infty$, this result implies the following $\epsilon$-rigidity result:

Corollary 1.3. Let $\left(M^{n}, g\right)$ be a complete Riemannian manifold whose metric is critical. Assume that $\left(M^{n}, g\right)$ satisfies the Sobolev inequality:

$$
\forall f \in C_{0}^{\infty}(M),\left(\int_{M}|f(y)|^{\frac{2 n}{n-2}} d \operatorname{vol}(y)\right)^{1-\frac{2}{n}} \leq A \int_{M}|d f(y)|^{2} d \operatorname{vol}(y) .
$$

If the curvature tensor satisfies

$$
A^{\frac{n}{2}} \int_{M}|\operatorname{Rm}(y)|^{\frac{n}{2}} d \operatorname{vol}_{g}(y)<\epsilon
$$

then $\left(M^{n}, g\right)$ is isometric to the Euclidean space $\mathbb{R}^{n}$.

In another paper [37], G. Tian and J. Viaclovsky were able to replace the hypothesis on the Sobolev inequality with a uniform lower bound on the volume growth of geodesic balls:

$$
\forall y \in B(x, r), \forall s \in(0, r): \operatorname{vol} B(y, s) \geq v s^{n}
$$

It is known that the Sobolev inequality implies such a uniform lower bound ([1] or [8]). The proof of this improvement used as a preliminary result the above $\epsilon$ regularity result (Theorem 1.2) and hence it relied on the intricate De Giorgi-NashMoser iteration scheme developed in [35] or [12]. Our idea leads to a direct proof of this improvement that does not use this iteration scheme ( $c f$. Theorem 3.2):

Theorem 1.4. There is a constant $\epsilon>0$ that only depends on $n$ and on the constant $\Upsilon$ appearing in the estimate (1.1) such that, if $(M, g)$ is a complete Riemannian manifold whose metric is critical and such that for some $x \in M$ and $r>0$, we have

i) $\forall y \in B\left(x, \frac{3}{4} r\right), \forall s \in(0, r / 4)$, $\operatorname{vol} B(y, s) \geq v s^{n}$

ii) $\int_{B(x, r)}|\mathrm{Rm}|^{\frac{n}{2}}(y) d y \leq \varepsilon v$ 
then

$$
\sup _{B\left(x, \frac{1}{2} r\right)}|\operatorname{Rm}| \leq \frac{16}{r^{2}}\left(\frac{1}{v \epsilon} \int_{B(x, r)}|\operatorname{Rm}|^{\frac{n}{2}}(y) d y\right)^{\frac{2}{n}} .
$$

Moreover we are able to give some $L^{p} \epsilon$-regularity/rigidity result, for instance we shall obtain the following:

Theorem B. There is a constant $\epsilon>0$ that only depends on $n, p$ and on the constant $\Upsilon$ appearing in the estimate (1.1) such that, when $\left(M^{n}, g\right)$ be a complete Riemannian manifold whose metric is critical and such that any geodesic ball $B \subset M$ (with radius $r(B)$ ) satisfies:

$$
\frac{r(B)^{2 p}}{\operatorname{vol} B} \int_{B}|\operatorname{Rm}(y)|^{p} d \operatorname{vol}_{g}(y)<\epsilon
$$

then the metric $g$ is flat: $\mathrm{Rm}=0$.

Our argument also leads to a new and direct proof of the following result of M. Anderson:

Theorem 1.5. There is a positive constant $\epsilon_{n}>0$ such that if $\left(M^{n}, g\right)$ is a complete Ricci flat manifold satisfying ${ }^{1}$ :

$$
\lim _{r \rightarrow \infty} \frac{\operatorname{vol} B(x, r)}{r^{n}} \geq \omega_{n}\left(1-\epsilon_{n}\right)
$$

then $\left(M^{n}, g\right)$ is isometric to the Euclidean space $\mathbb{R}^{n}$.

This result was used by Anderson to prove a $\epsilon$-regularity result based on volume growth for metrics with bounded Ricci curvature; for Einstein metrics, this result implies some uniform bound on the Riemann curvature tensor. In fact we obtain a new proof and a new formulation of this estimate:

Theorem C. There are constants $\epsilon(n)>0$ and $C(n)$ such that if $\left(M^{n}, g\right)$ is a complete Ricci flat manifold and $x \in M$ and $r>0$ are such that

$$
\operatorname{vol} B(x, r) \geq \omega_{n}\left(1-\epsilon_{n}\right) r^{n}
$$

then

$$
\sup _{B(x, r / 2)}|\mathrm{Rm}| \leq \frac{C(n)}{r^{2}} \sup _{y \in B\left(x, \frac{3}{4} r\right)}\left(\frac{\omega_{n} r^{n}-\operatorname{vol} B(y, r)}{r^{n}}\right)^{\frac{1}{4}} .
$$

Our idea is quite versatile and can be used to obtain other rigidity and regularity results. In a future work, we intend to consider applications of these ideas to the question of convergence of Einstein/critical metric in dimension $n>4$ in the spirit

${ }^{1} \omega_{n}$ is the volume of the unit Euclidean ball. 
of results of J. Cheeger, T. Colding, G. Tian [10] or of G. Tian and J. Viaclovsky [36]. What nowadays is missing is an answer to a question of M. Anderson ( $c f$. [2, Rem 2, p. 475] and G. Tian [34] about the geometry of Einstein/critical Riemannian manifolds with maximal volume growth and whose curvature satisfies some bound on:

$$
\sup _{r}\left(r^{4-n} \int_{B(x, r)}|\mathrm{Rm}|^{2}\right) .
$$

ACKnowledgements. I would like to thank E. Aubry, P. Castillon, R. Mazzeo, V. Minerbe for helpful discussions. Moreover, I thank the anonymous referee for very useful comments.

\section{Some Definitions and useful tools}

\subsection{Regular metrics}

Definition 2.1. We say that a Riemannian manifold $\left(M^{n}, g\right)$ is $(\Lambda, k)$-regular or that the Riemannian metric $g$ satisfies $(\Lambda, k)$-regularity estimates if for any $x \in M$ and any $r>0$ and $\varepsilon \in(0,1)$ such that

$$
\sup _{B(x, \varepsilon r)}|\mathrm{Rm}| \leq \frac{1}{r^{2}},
$$

then for all $j=1, \ldots, k$

$$
\sup _{B\left(x, \frac{1}{2} \varepsilon r\right)}\left|\nabla^{j} \mathrm{Rm}\right| \leq \frac{\Lambda}{(\varepsilon r)^{j} r^{2}} .
$$

\section{Remarks 2.2.}

a) The choice of half the radius in the estimate (2.1) is arbitrary, indeed it is easy to show that the $(\Lambda, k)$-regularity estimate implies the following: if for some $x \in M, r>0$ and $\varepsilon \in(0,1)$ we have

$$
\sup _{B(x, \varepsilon r)}|\mathrm{Rm}| \leq \frac{1}{r^{2}}
$$

then for all $\delta \in(0,1)$ and all $j=1, \ldots, k$, we have

$$
\sup _{B(x, \delta \varepsilon r)}\left|\nabla^{j} \mathrm{Rm}\right| \leq \frac{\Lambda}{\varepsilon^{j}((1-\delta) r)^{2+j}} .
$$


b) This condition of regularity is clearly invariant by scaling: if a metric $g$ satisfies $(\Lambda, k)$-regularity estimates then for any positive constant $h$, the metric $h^{2} g$ satisfies $(\Lambda, k)$-regularity estimates.

c) Hence, a metric $g$ satisfies $(\Lambda, k)$-regularity estimates if and only if for every positive constant $h$ the metric $g_{h}=h^{2} g$ satisfies the following estimates: for any $x \in M$ and any $\varepsilon \in(0,1)$ such that

$$
\sup _{B_{g_{h}}(x, 1)}\left|\mathrm{Rm}_{g_{h}}\right| \leq \varepsilon^{2},
$$

then for all $j=1, \ldots, k$

$$
\sup _{B_{g_{h}}\left(x, \frac{1}{2}\right)}\left|\nabla^{j} \operatorname{Rm}_{g_{h}}\right| \leq \Lambda \varepsilon^{2} .
$$

Sometimes, we will use a weaker assumption on the metric:

Definition 2.3. We say that a Riemannian manifold $\left(M^{n}, g\right)$ is weakly $(\Lambda, k)$-regular if if for any $x \in M$ and any $r>0$ such that

$$
\sup _{B(x, r)}|\mathrm{Rm}| \leq \frac{1}{r^{2}}
$$

then for all $j=1, \ldots, k$

$$
\sup _{B\left(x, \frac{r}{2}\right)}\left|\nabla^{j} \mathrm{Rm}\right| \leq \frac{\Lambda}{r^{j+2}} .
$$

\subsection{Examples of regular metrics}

\subsubsection{Einstein metrics and metrics with harmonic curvature}

When $\left(M^{n}, g\right)$ is Einstein

$$
\text { Ricci }_{g}=(n-1) \tau g
$$

then the curvature satisfies an elliptic equation

$$
\nabla^{*} \nabla \mathrm{Rm}+\mathcal{R}(\mathrm{Rm})=0
$$

where $\mathcal{R}$ is a certain action of the curvature operator on the space of curvature tensors. Indeed the Bianchi identities imply that

$$
d^{\nabla} \mathrm{Rm}=0
$$

and the fact that the Ricci curvature is parallel implies that the curvature tensor (viewed as a 2-forms valued in symmetric tensors) is coclosed:

$$
\left(d^{\nabla}\right)^{*} \mathrm{Rm}=0,
$$


hence the above equation (2.2) is a consequence of a Bochner formula ( $c f$. [4, Proposition 4.2])

$$
\left(d^{\nabla}\right)^{*} d^{\nabla}+d^{\nabla}\left(d^{\nabla}\right)^{*}=\nabla^{*} \nabla+\mathcal{R} .
$$

So that any harmonic Riemann tensor:

$$
\left(d^{\nabla}\right)^{*} \mathrm{Rm}=0
$$

satisfies the Bochner formula (2.2). This implies the following:

Proposition 2.4. If $\left(M^{n}, g\right)$ is a Riemannian manifold with harmonic curvature:

$$
\left(d^{\nabla}\right)^{*} \mathrm{Rm}=0
$$

then $\left(M^{n}, g\right)$ is $(\Lambda, k)$-regular for a constant $\Lambda$ that only depends on $n$ and $k$.

Proof. This regularity result can be proved with some rather classical elliptic regularity estimates, along the line of the proof of regularity of critical metrics (see the proof of Proposition 2.6). But we can also use less elaborate tools using only the maximum principle (following for instance the argumentation of W. Shi, [31, Section 7]).

Indeed assume that $g$ is a complete Riemannian metric with harmonic curvature. If we assume that on a geodesic ball $B(x, 1) \subset M$, and for some $\varepsilon \in(0,1)$, we have the following uniform bound on the curvature:

$$
\sup _{B(x, 1)}|\mathrm{Rm}| \leq \varepsilon^{2}
$$

Then the exponential map is a local diffeomorphism from the unit Euclidean ball $\mathbb{B}(0,1) \subset\left(T_{x} M, g_{x}\right)$ to $B(x, 1)$ :

$$
\exp _{x}: \mathbb{B}(0,1) \rightarrow B(x, 1)
$$

Then metric $\mathbf{g}=\exp _{x}^{*} g$ has also a harmonic curvature tensor and its curvature tensor is bounded by $\varepsilon^{2}$. We will prove the regularity estimate in the ball $\mathbb{B}(0,1)$ endowed with the metric $\mathbf{g}=\exp _{x}^{*} g$. Hence we work now on the Riemannian manifold $(\mathbb{B}(0,1), \mathbf{g})$. The Bochner's formulae imply that ${ }^{2}$ :

$$
\begin{aligned}
\Delta|\mathrm{Rm}|^{2} & \leq C(n) \epsilon^{2}|\mathrm{Rm}|^{2}-2|\nabla \mathrm{Rm}|^{2} \\
\text { and } \quad \Delta|\nabla \mathrm{Rm}|^{2} & \leq C(n) \epsilon^{2}|\nabla \mathrm{Rm}|^{2}-2\left|\nabla^{2} \mathrm{Rm}\right|^{2} .
\end{aligned}
$$

We define $v=\left(33 \epsilon^{4}+|\operatorname{Rm}|^{2}\right)|\nabla \operatorname{Rm}|^{2}$ and consider the function $\varphi=2 u-u^{2}$

${ }^{2} C(n)$ will be a constant that only depends on $n$ and that can vary from one estimate to another. 
where

$$
u(y)= \begin{cases}1 & \text { if }|y|^{2} \leq 1 / 2 \\ \left(3-4|y|^{2}\right)^{2} & \text { if } 1 / 2 \leq|y|^{2} \leq 3 / 4 \\ 0 & \text { if } 3 / 4 \leq|y|^{2}\end{cases}
$$

Then we have

$$
|\Delta \varphi| \leq C(n) \text { and }|d \varphi|^{2} \leq \varphi .
$$

Hence at a point where the function $\varphi v$ is maximal we have

$$
v d \varphi+\varphi d v=0 \text { and } 0 \leq \Delta(\varphi v) .
$$

Hence at such a point:

$$
\begin{aligned}
0 & \leq v \Delta \varphi-2\langle d \varphi, d v\rangle+\varphi \Delta v \\
& \leq v \Delta \varphi+2 \frac{|d \varphi|^{2}}{\varphi} v+\varphi \Delta v \\
& \leq C(n) v+\varphi \Delta v .
\end{aligned}
$$

A quick computation shows that

$$
\begin{aligned}
\Delta v \leq & \left(C(n) \epsilon^{2}|\mathrm{Rm}|^{2}-2|\nabla \mathrm{Rm}|^{2}\right)|\nabla \mathrm{Rm}|^{2} \\
& \quad+\left(33 \epsilon^{4}+|\mathrm{Rm}|^{2}\right)\left(C(n) \epsilon^{2}|\nabla \mathrm{Rm}|^{2}-2\left|\nabla^{2} \mathrm{Rm}\right|^{2}\right) \\
& \quad-2\left\langle d|\mathrm{Rm}|^{2}, d|\nabla \mathrm{Rm}|^{2}\right\rangle \\
\leq & C(n) \epsilon^{2} v-2|\nabla \mathrm{Rm}|^{4}-2\left(33 \epsilon^{4}+|\mathrm{Rm}|^{2}\right)\left|\nabla^{2} \mathrm{Rm}\right|^{2} \\
& \quad+8|\mathrm{Rm}||\nabla \mathrm{Rm}|^{2}\left|\nabla^{2} \mathrm{Rm}\right| \\
\leq & C(n) \epsilon^{2} v-|\nabla \mathrm{Rm}|^{4}-2\left(33 \epsilon^{4}+|\mathrm{Rm}|^{2}\right)\left|\nabla^{2} \mathrm{Rm}\right|^{2}+16|\mathrm{Rm}|^{2}\left|\nabla^{2} \mathrm{Rm}\right|^{2} \\
\leq & C(n) \epsilon^{2} v-|\nabla \mathrm{Rm}|^{4}-2\left(33 \epsilon^{4}+|\mathrm{Rm}|^{2}\right)\left|\nabla^{2} \mathrm{Rm}\right|^{2}+16 \varepsilon^{4}\left|\nabla^{2} \mathrm{Rm}\right|^{2} \\
\leq & C(n) \epsilon^{2} v-|\nabla \mathrm{Rm}|^{4} .
\end{aligned}
$$

Hence at a point where the function $\varphi v$ is maximal, we have

$$
0 \leq C(n) v+C(n) \epsilon^{2} \varphi v-\varphi|\nabla \mathrm{Rm}|^{4},
$$

so that we have at such a point:

$$
\varphi^{2} \frac{v^{2}}{\left(34 \varepsilon^{4}\right)^{2}} \leq \varphi^{2}|\nabla \mathrm{Rm}|^{4} \leq C(n) \varphi v .
$$

This estimate implies the following

$$
\sup _{\mathbb{B}(0,1)} \varphi v \leq C(n) \varepsilon^{8},
$$


and with the definition of $v=\left(33 \epsilon^{4}+|\mathrm{Rm}|^{2}\right)|\nabla \mathrm{Rm}|^{2}$, we get:

$$
\sup _{\mathbb{B}\left(0, \frac{1}{2}\right)}|\nabla \mathrm{Rm}|^{2} \leq C(n) \varepsilon^{4} .
$$

The estimate on the higher order covariant derivative of the Riemann tensor $\left|\nabla^{j} \mathrm{Rm}\right|$ can be obtained with the same argument using commutation rules between the covariant derivative $\nabla$ and the rough Laplacian $\nabla^{*} \nabla$.

We have already seen that Einstein metric have harmonic Riemann tensor; another example of metric with harmonic tensor are locally conformally flat metric with constant scalar curvature.

\subsubsection{Critical metrics}

As noticed by G. Tian and J. Viaclovsky [35], another large class of Riemannian metrics satisfies these regularity estimates:

Definition 2.5. We say that a Riemannian metric is critical if its Ricci tensor satisfies an Bochner's type equality:

$$
\nabla^{*} \nabla \operatorname{Ricci}_{g}+\mathcal{R}\left(\operatorname{Ricci}_{g}\right)=0
$$

where $\mathcal{R}$ is a linear action of the Riemann curvature tensor on the space of symmetric 2 tensors.

Proposition 2.6. A manifold $\left(M^{n}, g\right)$ endowed with a complete critical metric is is $(\Lambda, k)$-regular for a constant $\Lambda$ that only depends on $n$ and $k$ and on the Bochner formula (2.3).

Proof. The idea of the proof has already been sketched by X-X. Chen and B. Weber (cf. [12, page 1320]). First, using twice the Bianchi identities, we have (see: [4, formula 3.7]):

$$
\nabla^{*} \nabla \mathrm{Rm}+\mathcal{R}(\mathrm{Rm})=d^{\nabla}\left(d^{\nabla}\right)^{*} \mathrm{Rm}=-d^{\nabla}{\widehat{d^{\nabla} \mathrm{Ricci}_{g}}}
$$

where $d^{\nabla \operatorname{Ricci}_{g}}(X, Y, Z)=d^{\nabla} \operatorname{Ricci}_{g}(Y, Z, X)$. Now we can use the coupled elliptic system:

$$
\left\{\begin{array}{l}
\nabla^{*} \nabla \mathrm{Rm}^{+} \mathcal{R}(\mathrm{Rm})=-d^{\nabla} d^{\nabla \text { Ricci }_{g}} \\
\nabla^{*} \nabla \operatorname{Ricci}_{g}+\mathcal{R}\left(\text { Ricci }_{g}\right)=0
\end{array}\right.
$$

By scaling, we assume that on some geodesic ball $B(x, 1)$ and for some $\varepsilon \in(0,1)$, we have the following uniform bound on the curvature:

$$
\sup _{B(x, 1)}|\mathrm{Rm}| \leq \varepsilon^{2}
$$


Then the exponential map is a local diffeomorphism form the unit Euclidean ball $\mathbb{B}(0,1) \subset\left(T_{x} M, g_{x}\right)$ to $B(x, 1)$

$$
\exp _{x}: \mathbb{B}(0,1) \rightarrow B(x, 1) .
$$

Then metric $\mathbf{g}=\exp _{x}^{*} g$ is also critical and has its curvature tensor bounded by $\varepsilon^{2}$. We will prove the regularity estimate in the ball $\mathbb{B}(0,1)$ endowed with the metric $\mathbf{g}=\exp _{x}^{*} g$. Hence we work now on the Riemannian manifold $(\mathbb{B}(0,1), \mathbf{g})$ :

Moreover according to J. Jost and H. Karcher [14], M. Anderson [3, Remark 2.3i) ] there is a constant $\delta_{n}$ such that around each point $p \in \mathbb{B}(0,1 / 2)$ there is a harmonic chart on the ball of radius $\delta_{n}$

$$
\mathbf{x}: \mathbb{B}\left(p, \delta_{n}\right) \rightarrow \mathbb{R}^{n}
$$

such that the metric $\mathbf{x}_{*} \mathbf{g}$ has uniform $\mathcal{C}^{1, \alpha}$ and $W^{2,2 n}$ estimate.

Looking at the elliptic equation (2.3) in these coordinates implies that we have a uniform $W^{2,2 n}$ bound

$$
\|\operatorname{Ricci}\|_{W^{2,2 n}\left(\mathbb{B}\left(p, \delta_{n} / 2\right)\right)} \leq C(n)\|\operatorname{Rm}\|_{L^{2 n}\left(\mathbb{B}\left(p, \delta_{n}\right)\right)} \leq C(n) \varepsilon^{2} .
$$

So that we get an estimate

$$
\left\|\nabla^{2} \operatorname{Ricci}\right\|_{L^{2 n}\left(\mathbb{B}\left(p, \delta_{n} / 2\right)\right)} \leq C(n) \varepsilon^{2} .
$$

If we look now at the elliptic equation

$$
\nabla^{*} \nabla \mathrm{Rm}+\mathcal{R}(\mathrm{Rm})=-d^{\nabla} d^{\nabla \text { Ricci }_{g}}
$$

then we get similarly

$$
\begin{aligned}
\|\mathrm{Rm}\|_{W^{2,2 n}\left(\mathbb{B}\left(p, \delta_{n} / 4\right)\right)} & \leq C(n)\left[\|\operatorname{Rm}\|_{L^{2 n}\left(\mathbb{B}\left(p, \delta_{n} / 2\right)\right)}+\left\|\nabla^{2} \operatorname{Ricci}\right\|_{L^{2 n}\left(\mathbb{B}\left(p, \delta_{n} / 2\right)\right)}\right] \\
& \leq C(n) \varepsilon^{2} .
\end{aligned}
$$

In particular we have a uniform estimate on $\nabla \mathrm{Rm}$ on these balls $\mathbb{B}\left(p, \delta_{n} / 4\right)$,

$$
\sup _{\mathbb{B}\left(0, \frac{2+\delta_{n}}{4}\right)}|\nabla \mathrm{Rm}| \leq C(n) \varepsilon^{2} .
$$

These argument can be bootstrapped because a uniform bound on $\nabla^{j} \mathrm{Rm}, j=$ $0, \ldots k$ implies uniform $\mathcal{C}^{k+1, \alpha}$ and $W^{k+2,2 n}$ estimates on the metric $\mathbf{x}_{*} \mathbf{g}$ and these estimates on the metric imply a $W^{k, 2 n}$ estimate on the curvature tensor. 
Some example of critical metrics:

i) A Kähler metric with constant scalar curvature is critical. Indeed if $(M, \omega)$ is a Kähler manifold with Ricci form $\rho$. The Ricci form is closed of type $(1,1)$ and we have

$$
d^{*} \rho=-d^{c} \operatorname{Scal}_{g} .
$$

When the scalar curvature is constant, the Bochner formula on $(1,1)$ forms implies that

$$
0=\left(d d^{*}+d^{*} d\right) \rho=\nabla^{*} \nabla \rho+\mathcal{R}(\rho) .
$$

ii) Another important example is the case of Bach flat metric in dimension 4.

\subsection{The point selection lemma}

The following proposition can be found in [22, Appendix $\mathrm{H}]$ and is also known as the $1 / 4$-almost maximum lemma (see the $\lambda$-maximum lemma in $[17$, p. 256]). A version of this point selection lemma has also been used and proved by R. Schoen (see [28, proof of Theorem 2.2]).

Proposition 2.7. Assume that $\varphi: X \rightarrow \mathbb{R}_{+}$is a continuous function on a complete locally compact metric space $(X, d)$. If for some $x_{0} \in X$ and $r>0$ we have

$$
\varphi\left(x_{0}\right) \geq \frac{1}{r^{2}}
$$

then for any $A>0$ there is a point $\bar{x} \in B\left(x_{0}, 2 A r\right)$ such that

$$
\varphi(\bar{x}) \geq \frac{1}{r^{2}}
$$

and

$$
\forall z \in B\left(\bar{x}, A \varphi(\bar{x})^{-1 / 2}\right), \varphi(z) \leq 4 \varphi(\bar{x}) .
$$

Proof. Starting from $x_{0}$ we build inductively a sequence $x_{0}, x_{1} \ldots$

If $x_{l}$ is such that on $B\left(x_{0}, d\left(x_{0}, x_{l}\right)+A \varphi\left(x_{l}\right)^{-1 / 2}\right)$

$$
\varphi \leq 4 \varphi\left(x_{l}\right)
$$

then we define

$$
x_{l+1}=x_{l} .
$$

If it is not the case then we can find $x_{l+1}$ such that

$$
d\left(x_{0}, x_{l+1}\right) \leq d\left(x_{0}, x_{l}\right)+\frac{A}{\sqrt{\varphi\left(x_{l}\right)}}
$$

and

$$
\varphi\left(x_{l+1}\right) \geq 4 \varphi\left(x_{l}\right)
$$


If the points $x_{0}, x_{1}, \ldots, x_{N}$ are distincts then we get for $l \in\{0, \ldots, N\}$ :

$$
\varphi\left(x_{l}\right) \geq 4^{l} \varphi\left(x_{0}\right)
$$

and

$$
d\left(x_{0}, x_{l}\right) \leq \sum_{k=0}^{l-1} \frac{A}{\sqrt{\varphi\left(x_{k}\right)}} \leq 2 A r .
$$

As $\varphi$ is continuous and $B\left(x_{0}, 2 A r\right)$ compact, the sequence must stabilize.

Remark 2.8. We only need the fact that $\varphi$ is bounded on the ball $B(x, 2 A r)$.

\section{Some $\epsilon$-rigidity and regularity results}

\section{1. $\epsilon$-quadratic decay}

Theorem 3.1. Let $(M, g)$ be a complete non-compact Riemannian manifold whose metric is weakly $(\Lambda, 1)$-regular ( where $\Lambda \geq 1)$. Let $\epsilon=\frac{1}{6 \Lambda}$. If for some fixed point $o \in M$ we have:

$$
\forall y \in M, \quad|\operatorname{Rm}(y)| \leq \frac{\epsilon^{2}}{d(o, y)^{2}}
$$

then the metric $\mathrm{g}$ is flat: $\mathrm{Rm}=0$.

Proof. If the curvature does not vanish identically, then our hypothesis implies that we can find a point $x \in M$ where the curvature reached its maximum, in particular:

$$
|\operatorname{Rm}(x)|=\frac{1}{r^{2}} \text { and } \sup _{B(x, r)}|\operatorname{Rm}| \leq \frac{1}{r^{2}} .
$$

By $(\Lambda, 1)$-regularity, we know that

$$
\sup _{B(x, r / 2)}|\nabla \mathrm{Rm}| \leq \Lambda \frac{1}{r^{3}} .
$$

In particular, for $\delta=1 /(2 \Lambda)$, we have for $y \in B(x, \delta r)$ :

$$
|\operatorname{Rm}(y)| \geq|\operatorname{Rm}(x)|-\delta r \Lambda \frac{1}{r^{3}} \geq \frac{1}{2}|\operatorname{Rm}(x)|=\frac{1}{2 r^{2}} .
$$

We have supposed

$$
|\operatorname{Rm}(x)| \leq \frac{\epsilon^{2}}{d(o, x)^{2}}
$$

hence

$$
d(o, x) \leq \epsilon r,
$$


and when $y \in \partial B(x, \delta r)$, we have $d(o, y) \geq d(y, x)-d(o, x) \geq \delta r-\epsilon r$ and

$$
\frac{1}{2 r^{2}} \leq|\operatorname{Rm}|(y) \leq \frac{\epsilon^{2}}{d(o, y)^{2}} \leq \frac{\epsilon^{2}}{(\delta-\epsilon)^{2} r^{2}} .
$$

Our choice of $\delta=3 \epsilon$ implies that

$$
\frac{\epsilon^{2}}{(\delta-\epsilon)^{2}}=\frac{1}{4}
$$

hence the result.

The curvature of an Einstein metric with zero scalar curvature is harmonic, hence by Proposition (2.4) an Einstein metric with zero scalar curvature is $(\Lambda(n), 1)$-regular for some constant $\Lambda(n)$ that only depends on the dimension $n$. So that Theorem A follows from the above Theorem 3.1.

\section{2. $L^{\frac{n}{2}} \epsilon$-regularity}

Theorem 3.2. Let $(M, g)$ be a complete Riemannian manifold whose metric is $(\Lambda, 1)$-regular for some $\Lambda \geq 1$. There is a constant $\epsilon(\Lambda, n)>0$ such that if for some $x \in M$ and $r>0$ we have

i) $\forall y \in B\left(x, \frac{3}{4} r\right), \forall s \in(0, r / 4)$, vol $B(y, s) \geq v s^{n}$

ii) $\int_{B(x, r)}|\operatorname{Rm}|^{\frac{n}{2}}(y) d y \leq \varepsilon(\Lambda, n) v$,

then

$$
\sup _{B\left(x, \frac{1}{2} r\right)}|\operatorname{Rm}| \leq \frac{16}{r^{2}}\left(\frac{1}{v \epsilon(\Lambda, n)} \int_{B(x, r)}|\operatorname{Rm}|^{\frac{n}{2}}(y) d y\right)^{\frac{2}{n}} .
$$

Proof. Assume that there is a point $z \in B\left(x, \frac{1}{2} r\right)$ such that

$$
|\operatorname{Rm}|(z) \geq \frac{\mu^{2}}{r^{2}}
$$

where $\mu \in(0,4]$. By the point selection lemma (with $A=\mu / 8$ ), we find a point $y \in B\left(z, \frac{1}{4} r\right) \subset B\left(x, \frac{3}{4} r\right)$ such that

$$
|\operatorname{Rm}(y)|=\frac{1}{\rho^{2}} \geq \frac{\mu^{2}}{r^{2}}
$$

and

$$
\sup _{B\left(y, 2 A\left(\frac{\rho}{2}\right)\right)}|\mathrm{Rm}| \leq \frac{4}{\rho^{2}} .
$$


By $(\Lambda, 1)$-regularity, we get

$$
\sup _{B(y, A \rho / 2)}|\nabla \mathrm{Rm}| \leq \Lambda \frac{8}{2 A \rho^{3}}=\frac{4 \Lambda}{A \rho^{3}} .
$$

As in the proof of Theorem 3.1, if we let

$$
\delta=\frac{A}{8 \Lambda}=\frac{\mu}{64 \Lambda}
$$

then on the ball $B(y, \delta \rho)$ :

$$
|\operatorname{Rm}| \geq \frac{1}{2 \rho^{2}}
$$

Hence we get

$$
\begin{aligned}
\int_{B(x, r)}|\operatorname{Rm}|^{\frac{n}{2}}(\sigma) d \sigma & \geq \int_{B(y, \delta \rho)}|\operatorname{Rm}|^{\frac{n}{2}}(\sigma) d \sigma \\
& \geq \frac{\operatorname{vol} B(y, \delta \rho)}{2^{\frac{n}{2}} \rho^{n}} \\
& \geq v\left(\frac{\delta}{\sqrt{2}}\right)^{n} .
\end{aligned}
$$

For

$$
\epsilon(\Lambda, n)=\left(\frac{1}{16 \Lambda \sqrt{2}}\right)^{n}
$$

we get that when $\int_{B(x, r)}|\operatorname{Rm}|^{\frac{n}{2}}(y) d y \leq \varepsilon(\Lambda, n) v$, we can not find a point $z \in$ $B\left(x, \frac{1}{2} r\right)$ such that

$$
|\operatorname{Rm}|(z) \geq \frac{16}{r^{2}} .
$$

Moreover when $z \in B\left(x, \frac{1}{2} r\right)$ then for $\mu^{2}=r^{2}|\operatorname{Rm}|(z)$ we get:

$$
v \epsilon(\Lambda, n)\left(\frac{\mu}{4}\right)^{n}=v\left(\frac{\mu}{64 \sqrt{2} \Lambda}\right)^{n} \leq \int_{B(x, r)}|\operatorname{Rm}|^{\frac{n}{2}}(\sigma) d \sigma .
$$

\section{Remarks 3.3.}

i) For Einstein manifolds, this result is due to M. Anderson ([2]): assume that

$$
\operatorname{Ricci}_{g}=(n-1) \tau g
$$

and denote by $V_{\tau}(r)$ the volume of a geodesic ball of radius $r$ in the simply connected complete Riemannian $n$-manifold with constant sectional curvature 
$\tau$, then the Bishop-Gromov inequality implies that for $y \in B\left(x, \frac{3}{4} r\right)$ and $s \in$ $(0, r / 4)$ we have:

$$
\operatorname{vol} B(y, s) \geq \frac{V_{\tau}(s)}{V_{\tau}(2 r)} \operatorname{vol} B(y, 2 r) \geq \frac{V_{\tau}(s)}{V_{\tau}(2 r)} \operatorname{vol} B(x, r) .
$$

Hence when $|\tau| r^{2} \leq 1$, our proof of Theorem 3.2 shows that the above hypothesis i) and ii) can be gathered in a single one:

$$
\frac{V_{\tau}(r)}{\operatorname{vol} B(x, r)} \int_{B(x, r)}|\operatorname{Rm}|^{\frac{n}{2}}(\sigma) d \sigma \leq \epsilon(n) .
$$

ii) For critical metrics and in dimension 4 , this result has been also proven G.Tian and J.Viaclovsky ([37, Theorem 1.2]). In fact, this result was a refinement of an earlier result in ([35, Theorem 3.1]) where the hypothesis i) was replaced by a Sobolev inequality:

$$
\forall \varphi \in C_{0}^{\infty}(B(x, r)), \quad\|\varphi\|_{L^{\frac{2 n}{n-2}}} \leq A\|d \varphi\|_{L^{2}} .
$$

And according to ([1] or [8]), such a Sobolev inequality implies a lower bound on the volume on geodesic ball: if $B \subset B(x, r)$ is a geodesic ball of radius $r(B)$ then

$$
\operatorname{vol} B \geq C(n)\left(\frac{r(B)}{A}\right)^{n} .
$$

It should also be noticed that the main argument in the proof of the result of G.Tian and J.Viaclovsky was also a point selection lemma that relies a priori to the $\epsilon$-regularity result on [35], that is the proof relies on an intricate De Giorgi-Moser-Nash iteration scheme argument. The results of G. Tian and J. Viaclovsky has been extended by X-X. Chen and B. Weber ([12]) in two directions: for extremal Kähler metrics and in dimension $n>4$. Now from the proof of ([37, Proposition 3.1]), it is clear that the $\epsilon$-regularity result of X$\mathrm{X}$. Chen and B. Weber (see [12, Theorem 4.6]) implies the above $\epsilon$-regularity result. But our proof is shorter and does not rely on De Giorgi-Moser-Nash iteration scheme argument but on quite classical elliptic estimates.

iii) Eventually, it should be noticed that if we assume that the metric is $(\Lambda, k)$ regular, then we get estimates on the covariant derivative of the Riemann tensor $\nabla^{j} \mathrm{Rm}, j=1, \ldots, k$.

Letting the radius $r$ going to $\infty$, this result also implies a $\epsilon-L^{\frac{n}{2}}$ rigidity result:

Corollary 3.4. Let $(M, g)$ is a complete Riemannian manifold whose metric is $(\Lambda, 1)$-regular for some $\Lambda \geq 1$. Assume that:

i) $\forall x \in M$ and $\forall r>0, \operatorname{vol} B(x, r) \geq v r^{n}$

ii) $\int_{M}|\operatorname{Rm}|^{\frac{n}{2}}(y) d y \leq \varepsilon(\Lambda, n) v$.

Then

$$
\mathrm{Rm}=0 .
$$




\section{3. $\epsilon-L^{p}$-regularity}

The above argument can be extended to other $L^{p}$ estimates on the curvature:

Theorem 3.5. Let $(M, g)$ be a complete Riemannian manifold whose metric is $(\Lambda, 1)$-regular for some $\Lambda \geq 1$. Let $p>0$. For any $x \in M$ and $r>0$ we let $\mathcal{B}$ be the collection of geodesic balls $B$ of radius $r(B)$ included in $B(x, r)$.

$$
\mathcal{M}(x, r):=\sup _{B \in \mathcal{B}}\left(\frac{r(B)^{2 p}}{\operatorname{vol} B} \int_{B}|\operatorname{Rm}|^{p}\right)^{\frac{1}{p}} .
$$

There is a constant $\epsilon(\Lambda, p)>0$ such that if for some $x \in M$ and $r>0$ we have

$$
\mathcal{M}(x) \leq \epsilon(\Lambda, p)
$$

then

$$
\sup _{B\left(x, \frac{1}{2} r\right)}|\operatorname{Rm}| \leq \frac{16}{\epsilon(\Lambda, p) r^{2}} \mathcal{M}(x, r) .
$$

And we also get the following $\epsilon-L^{p}$ rigidity result:

Corollary 3.6. Let $(M, g)$ is a complete Riemannian manifold whose metric is $(\Lambda, 1)$-regular for some $\Lambda \geq 1$. Assume that: $\forall x \in M$ and $\forall r>0$ :

$$
\frac{r^{2 p}}{\operatorname{vol} B(x, r)} \int_{B(x, r)}|\operatorname{Rm}|^{p}(y) d y \leq \varepsilon(\Lambda, p)^{p} .
$$

Then

$$
\mathrm{Rm}=0 .
$$

It is also clear that these results together with [35, Theorem 4.1] give some conditions that imply finiteness of the number of ends and that each end is ALE of order 0 , but we prefer to refrain from stating it.

\section{Almost maximal volume growth}

With the point selection lemma, we are going to give an alternative proof of the following (slightly improved) result of Anderson [3]:

Theorem 4.1. There are constant $\epsilon(n)>0$ and $C(n)$ such that if $\left(M^{n}, g\right)$ is a complete Ricci flat manifold and $x \in M$ and $r>0$ are such that ${ }^{3}$

$$
\operatorname{vol} B(x, r) \geq \omega_{n}\left(1-\epsilon_{n}\right) r^{n}
$$

$3 \omega_{n}$ is the volume of the unit Euclidean ball. 
then

$$
\sup _{B(x, r / 2)}|\mathrm{Rm}| \leq \frac{C(n)}{r^{2}} \sup _{y \in B\left(x, \frac{3}{4} r\right)}\left(\frac{\omega_{n} r^{n}-\operatorname{vol} B(y, r)}{r^{n}}\right)^{\frac{1}{4}} .
$$

This theorem has the following corollary

Corollary 4.2. If $\left(M^{n}, g\right)$ is a complete Ricci flat manifold such that

$$
\lim _{r \rightarrow \infty} \frac{\operatorname{vol} B(x, r)}{r^{n}} \geq \omega_{n}\left(1-\epsilon_{n}\right)
$$

then $\left(M^{n}, g\right)$ is isometric to the Euclidean space $\mathbb{R}^{n}$.

Anderson has shown first Corollary 4.2 with an argument by contradiction and then he deduced (still by contradiction) an estimate for the $\mathcal{C}^{1, \alpha}$-harmonic radius when the volume of the geodesic ball is almost maximal under a uniform bound on the Ricci curvature. When the manifold is Einstein, the elliptic regularity of the Einstein equation implies a bound on the curvature. For Einstein metrics, our curvature estimate is more precise. Here we are going to show Theorem 4.1, the Corollary 4.2 is then straightforward.

Proof. Again assume that there is a point $z \in B(x, r / 2)$ such that

$$
|\operatorname{Rm}|(z) \geq \frac{\mu^{2}}{r^{2}}
$$

where $\mu \in(0,4]$. By the point selection lemma (with $A=\mu / 8$ ) we find a point $y \in B\left(z, \frac{1}{4} r\right) \subset B\left(x, \frac{3}{4} r\right)$ such that

$$
|\operatorname{Rm}(y)|=\frac{1}{\rho^{2}} \geq \frac{\mu^{2}}{r^{2}}
$$

and

$$
\sup _{B\left(y, 2 A\left(\frac{\rho}{2}\right)\right)}|\mathrm{Rm}| \leq \frac{4}{\rho^{2}} .
$$

By $(\Lambda, 7)$-regularity, we get for $j=1, \ldots, 7$ :

$$
\sup _{B(y, \mu \rho / 16)}\left|\nabla^{j} \mathrm{Rm}\right| \leq \frac{C(n)}{(\mu \rho)^{j} \rho^{2}} .
$$

According to A. Gray and L. Vanhecke, we know the asymptotic expansion of the volume of geodesic balls [15], [16, Theorem 3.3]:

$$
\operatorname{vol} B(y, r)=\omega_{n} r^{n}\left(1-\frac{1}{120(n+2)(n+4)}|\operatorname{Rm}(y)|^{2} r^{4}+O\left(r^{6}\right)\right) \text {. }
$$




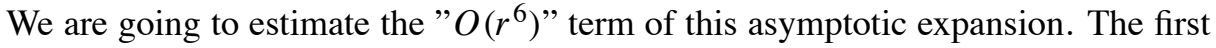
step is to remark that if $\mathbb{B}(s)$ is the Euclidean ball of radius $s$ in $\left(T_{y} M, g_{y}\right)$ then

$$
\exp _{y}: \mathbb{B}(A \rho) \rightarrow B(y, A \rho)
$$

is an immersion, hence for $\bar{g}=\exp _{y}^{*} g$, we get for all $r \leq A \rho$ :

$$
\operatorname{vol} B(y, r) \leq \operatorname{vol}_{\bar{g}} \mathbb{B}(\rho) .
$$

Estimate (4.1) and the Jacobi equation imply that if $t \mapsto J(t)$ is a Jacobi field along the geodesic $t \mapsto \exp _{y}(t v)$ with $|v|=1, J(0)=0$ and $\left|J^{\prime}(0)\right|=1$ then for all $t \in[0, \mu \rho / 16]$ and $l \in\{0, \ldots, 7\}$ :

$$
\left|\frac{d^{l}}{d t^{l}} J(t)\right| \leq B_{n}|\operatorname{Rm}(y)|(\mu \rho)^{3-l} .
$$

Then Gray and Vanhecke's computation leads to

$\forall s \in(0, \mu \rho / 16), \operatorname{vol}_{\bar{g}} \mathbb{B}(s)=\omega_{n} s^{n}\left(1-\frac{1}{120(n+2)(n+4)}|R m(y)|^{2} s^{4}+\delta(s)\right)$

where for some constant $D_{n}>1$ depending only on the dimension $n$ :

$$
|\delta(s)| \leq D_{n} s^{6}|\operatorname{Rm}(y)|(\mu \rho)^{-4} .
$$

We choose $s=\eta_{n} \mu^{2} \rho$ such that

$$
D_{n} s^{6}|R m(y)|(\mu \rho)^{-4}=\frac{1}{240(n+2)(n+4)}|\operatorname{Rm}(y)|^{2} s^{4}
$$

i.e.,

$$
\eta_{n}^{2}=\frac{1}{240(n+2)(n+4) D_{n}} .
$$

Then we get for $\sigma=\eta_{n} \mu^{2} \rho$

$$
\frac{\operatorname{vol} B(y, r)}{r^{n}} \leq \frac{\operatorname{vol} B(y, \sigma)}{\sigma^{n}} \leq \frac{\operatorname{vol}_{\bar{g}} \mathbb{B}(\sigma)}{\sigma^{n}} \leq \omega_{n}\left(1-\frac{\eta_{n}^{4} \mu^{8}}{240(n+2)(n+4)}\right) .
$$

\subsection{A sphere theorem}

With the same idea, we can give a direct proof of the following result.

Theorem 4.3. There is a $\varepsilon_{n}>0$ such that if $\left(M^{n}, g\right)$ is closed Einstein manifold with positive scalar curvature:

$$
\text { Ricci }_{g}=(n-1) g
$$

and

$$
\frac{\operatorname{vol}(M, g)}{\operatorname{vol} \mathbb{S}^{n}} \geq 1-\varepsilon_{n}
$$

then $(M, g)$ is isometric to the round sphere $\mathbb{S}^{n}$. 
Perhaps, there is a nice optimal volume pinching for Einstein metrics with positive scalar curvature; a nice result in this direction has been proved by M. Gursky ([18]); it states that any non-standard Einstein metric $g$ on the sphere $\mathbb{S}^{4}$ must satisfy

$$
\frac{\operatorname{vol}\left(\mathbb{S}^{4}, g\right)}{\operatorname{vol} \mathbb{S}^{4}} \leq \frac{1}{3}
$$

The same proof will also prove a local version of this result: for $r \in[0, \pi]$, we denote by $V_{1}(r)$ the volume of a geodesic ball in $\mathbb{S}^{n}$ :

$$
V_{1}(r)=\operatorname{vol}\left(\mathbb{S}^{n-1}\right) \int_{0}^{r}(\sin (t))^{n-1} d t .
$$

Theorem 4.4. There is a $\varepsilon_{n}>0$ such that if $\left(M^{n}, g\right)$ is closed Einstein manifold with positive scalar curvature:

$$
\operatorname{Ricci}_{g}=(n-1) g
$$

and such that for some $r \in(0, \pi]$ and all $x \in M$ :

$$
\frac{\operatorname{vol}(B(x, r))}{V_{1}(r)} \geq 1-\varepsilon_{n} r^{4}
$$

then $(M, g)$ has constant sectional curvature.

These theorems are consequences of a result of M. Anderson and of the isolation of the round metric amongst Einstein metrics. Indeed, a consequence of Anderson's result ([3, Theorem 1.2]) is the following:

For $\delta>0$, we can choose $\epsilon(n, \delta)>0$ such that the hypothesis

$$
\operatorname{Ricci}_{g}=(n-1) g \text { and } \frac{\operatorname{vol}(M, g)}{\operatorname{vol} \mathbb{S}^{n}} \geq 1-\varepsilon(n, \delta)
$$

implies that the sectional curvature of $g$ are in an interval $(1-\delta, 1+\delta)$. Now according to $[6,7,19,23]$, we know that a Einstein metric with sectional curvature in the interval $\left(\frac{1}{2}, 2\right)$ has constant sectional curvature. If we do not care about the optimal value of the pinching condition such a rigidity result can be easily proven with the maximum principle.

Indeed the Weyl tensor $\mathrm{W}$ of an Einstein metric satisfies a Bochner formula ([4, Proposition 4.2], [32]):

$$
\nabla^{*} \nabla \mathrm{W}+\frac{2 \mathrm{Scal}_{g}}{n} \mathrm{~W}=\mathrm{W} * \mathrm{~W}
$$

where $\mathrm{W} * \mathrm{~W}$ is a quadratic expression in the Weyl tensor. Hence if $\mathrm{Ricci}_{g}=$ $(n-1) g$, we obtain that the length of the Weyl tensor satisfies:

$$
\Delta|\mathrm{W}|^{2}+4(n-1)|\mathrm{W}|^{2}=2\langle\mathrm{~W}, \mathrm{~W} * \mathrm{~W}\rangle-2|\nabla \mathrm{W}|^{2} .
$$


Hence at a point where the length of the Weyl tensor reaches its maximum, we have:

$$
4(n-1)|\mathrm{W}|^{2} \leq \Delta|\mathrm{W}|^{2}+4(n-1)|\mathrm{W}|^{2}=2\langle\mathrm{~W}, \mathrm{~W} * \mathrm{~W}\rangle \leq c(n)|\mathrm{W}|^{3} .
$$

Hence either $\mathrm{W}=0$ or $\max _{x \in M}|\mathrm{~W}(x)| \geq \frac{2(n-1)}{c(n)}$.

Proof. We use again the same idea to prove the above theorems. Assume that $\left(M^{n}, g\right)$ is a closed Einstein manifold with positive scalar curvature:

$$
\text { Ricci }_{g}=(n-1) g
$$

and that the sectional curvature of $g$ are not constant; then we know that

$$
\max _{M}|\mathrm{~W}| \geq \frac{2(n-1)}{c(n)} .
$$

Let $x \in M$ be a point where this maximum is reached:

$$
\frac{1}{\rho^{2}}=|\mathrm{W}(x)|=\max _{M}|\mathrm{~W}| .
$$

By regularity, we obtain estimates on all the covariant derivatives of the Weyl tensor: for $j \in\{1, \ldots, 7\}$

$$
\max _{M}\left|\nabla^{j} \mathrm{~W}\right| \leq C(n) \frac{1}{\rho^{j}}
$$

(recall that the diameter of $M$ is bounded by $\pi$ and that $\rho^{2} \leq \frac{2(n-1)}{c(n)}$ ).

The same argument using the computations of Gray and Vanhecke shows that for some constant $\delta_{n}>0$ and for all $s \in\left(0, \delta_{n} \rho\right)$ :

$$
\frac{\operatorname{vol}(B(x, s))}{V_{1}(s)} \leq 1-\frac{1}{240(n+2)(n+4)}\left(\frac{s}{\rho}\right)^{4} .
$$

Then the Bishop-Gromov comparison principle implies then that:

$$
\frac{\operatorname{vol}(M, g)}{\operatorname{vol} \mathbb{S}^{n}}=\frac{\operatorname{vol}(B(x, \pi))}{V_{1}(\pi)} \leq \frac{\operatorname{vol}\left(B\left(x, \delta_{n} \rho\right)\right)}{V_{1}\left(\delta_{n} \rho\right)} \leq 1-\frac{\delta_{n}^{4}}{240(n+2)(n+4)} .
$$

It also implies that for $r \in\left(\delta_{n} \rho, \pi\right]$

$$
\frac{\operatorname{vol}(B(x, r))}{V_{1}(r)} \leq 1-\frac{\delta_{n}^{4}}{240(n+2)(n+4)} \leq 1-\frac{\delta_{n}^{4} r^{4}}{240(n+2)(n+4) \pi^{4}}
$$

and because $\rho^{2} \leq \frac{2(n-1)}{c(n)}$, we have a constant $\eta_{n}$ such that for all $r \in(0, \pi]$ :

$$
\frac{\operatorname{vol}(B(x, r))}{V_{1}(r)} \leq 1-\eta_{n} r^{4}
$$




\subsection{Another rigidity result}

The same argument can be used to prove a volume rigidity result when the scalar curvature is zero and when the second term in the asymptotic expansion in the volume of geodesic balls has a definite sign:

Theorem 4.5. There is a constant $\varepsilon_{n}>0$, such that when $\left(M^{n}, g\right)$ is a complete locally conformally flat manifold with zero scalar curvature of dimension $n \geq 4$ such that for some $v>0$ :

$$
\forall x \in M, \forall r>0 \quad: v r^{n} \leq \operatorname{vol} B(x, r) \leq \omega_{n} r^{n}\left(1+\varepsilon_{n} v^{4}\right)
$$

then $\left(M^{n}, g\right)$ is isometric to the Euclidean space $\mathbb{R}^{n}$.

Proof. Indeed at a 4-almost maximal point of the length of the Riemann curvature tensor, we have

$$
|\operatorname{Rm}(x)|=\frac{1}{\rho^{2}} \text { and } \max _{B(x, \rho)}|\mathrm{Rm}| \leq \frac{4}{\rho^{2}} .
$$

Because vol $B(x, r) \geq v r^{n}$, Cheeger's estimate of the injectivity radius ([9], [11, Theorem 4.2]) implies that the injectivity radius at $x$ is bounded from below:

$$
\operatorname{inj}_{x} \geq \eta_{n} v \rho .
$$

Again if we denote by $\mathbb{B}(s)$ the Euclidean ball of radius $s$ in $\left(T_{x} M, g_{x}\right)$ then

$$
\exp _{x}: \mathbb{B}\left(\eta_{n} v \rho\right) \rightarrow B\left(y, \eta_{n} v \rho\right)
$$

is a diffeomorphism (note that our hypothesis implies in particular that $v \leq \omega_{n}$ ). Hence for $\mathbf{g}=\exp _{c}^{*} g$, we get for all $r \leq \eta_{n} v \rho$ :

$$
\operatorname{vol} B(y, r)=\operatorname{vol}_{\mathbf{g}} \mathbb{B}(r) .
$$

When the metric is locally conformally flat with zero scalar curvature, Gray and Vanhecke's computation gives

$$
\operatorname{vol} B(x, r)=\omega_{n} r^{n}\left(1+\frac{2 n-7}{90\left(n^{2}-4\right)(n+4)}\left|\operatorname{Ricci}_{g}(x)\right|^{2} r^{4}+O\left(r^{6}\right)\right) .
$$

The same arguments imply that for some $\delta_{n}>0$ and $\varepsilon_{n}>0$, we have for $s=\delta_{n} v \rho$

$$
\operatorname{vol} B(x, s) \geq \omega_{n} s^{n}\left(1+v^{4} \varepsilon_{n}\right) \text {. }
$$

Remark 4.6. Using [16, Corollary 3.4] in dimension 3, and because a control of the first seven derivatives of the curvature tensor is enough to estimate the remainder terms in the volume expansion of geodesic balls, the same proof furnishes that there is a $\varepsilon(\Lambda)>0$ such that if $(M, g)$ is complete $(\Lambda, 7)$-regular 3-manifold with zero scalar curvature such that

$$
\forall x \in M, \forall r \geq 0: \operatorname{vol} B(x, r) \geq \omega_{n} r^{3}(1-\varepsilon),
$$

then $(M, g)$ is isometric to the Euclidean space $\mathbb{R}^{3}$. 


\section{References}

[1] K. AKUTAGAWA, Yamabe metrics of positive scalar curvature and conformally flat manifolds, Differential Geom. Appl. 4 (1994), 239-258.

[2] M. ANDERSON, Ricci curvature bounds and Einstein metrics on compact manifolds, J. Amer. Math. Soc. 2 (1989), 455-490.

[3] M. ANDERSON, Convergence and rigidity of manifolds under Ricci curvature bounds, Invent. Math. 102 (1990), 429-445.

[4] J.-P. Bourguignon, Les variétés de dimension 4 à signature non nulle dont la courbure est harmonique sont d'Einstein, Invent. Math. 63 (1981), 263-286.

[5] P. BÉRARD, Remarques sur l'équation de J. Simons, In: "Differential Geometry", B. Lawson and K. Tenenblat (eds.), Pitman Monogr. Surveys Pure Appl. Math. 52, Longman Sci. Tech., Harlow, 1991, 47-57.

[6] C. BÖHM and B. WILKING, Manifolds with positive curvature operators are space forms, Ann. of Math. 167 (2008), 1079-1097.

[7] S. BRENDLE, Einstein manifolds with nonnegative isotropic curvature are locally symmetric, Duke Math J. 151 (2010), 1-21.

[8] G. CARRON, Inégalités isopérimétriques de Faber-Krahn et conséquences, In: "Actes de la Table Ronde de Géométrie Différentielle (Luminy, 1992)", Vol. 1 of Sémin. Congr., Paris: Soc. Math. France 1996, 205-232.

[9] J. CheEger, Finiteness theorems for Riemannian manifolds, Amer. J. Math. 92 (1970), 61-94.

[10] J. CHEEger, T. Colding and G. TIAN, On the singularities of spaces with bounded Ricci curvature, Geom. Funct. Anal. 12 (2002), 873-914.

[11] J. CHEEgER, M. GROMOV and M. TAYLOR, Finite propagation speed, kernel estimates for functions of the Laplace operator, and the geometry of complete Riemannian manifolds, J. Differential Geom. 17 (1982), 15-53.

[12] X. CHEN and B. WeBER, Moduli spaces of critical Riemanian metrics with $L^{\frac{n}{2}}$-norm curvature bounds, Adv. Math. 226 (2010), 1307-1330.

[13] Y. CHU, A rigidity theorem for complete noncompact Bach-flat manifolds, J. Geom. Phys. 61 (2011), 516-521.

[14] J. Jost and H. KarCher, Geom. Meth. zur gewinnung für harmonische Abbildung, Manuscripta Math. 40 (1982), 27-77.

[15] A. GRAY, The volume of a small geodesic ball of a Riemannian manifold, Michigan Math. J. 20 (1973), 329-344.

[16] A. GRAY and L. VANHECKE, Riemannian geometry as determined by the volumes of small geodesic balls, Acta Math. 142 (1979), 157-198.

[17] M. Gromov, Foliated Plateau problem, part II: harmonic maps of foliations, Geom. Funct. Anal. 1 (1991), 253-320.

[18] M. GURSKY, Four-manifolds with $\delta W^{+}=0$ and Einstein constants of the sphere, Math. Ann. 318 (2000), 417-431.

[19] G. HUISKEN, Ricci deformation of the metric on a Riemannian manifold, J. Differential Geom. 21 (1985), 47-62.

[20] M. IтOH and H. SATOH, of the Weyl conformal tensor for Einstein manifolds, Proc. Japan Acad. A 78 (2002), 140-142.

[21] S. KIM, Rigidity of noncompact complete Bach-flat manifolds, J. Geom. Phys. 60 (2010), 637-642.

[22] B. Kleiner and J. LotT, Notes on Perelman's papers, Geom. Topol. 12 (2008), 25872855.

[23] C. MARGERIN, A sharp characterization of the smooth 4-sphere in curvature terms, Comm. Anal. Geom. 6 (1998), 21-65.

[24] V. Minerbe, Weighted Sobolev inequalities and Ricci flat manifolds, Geom. Funct. Anal. 18 (2009), 1696-1749. 
[25] L. NI, Gap theorems for minimal submanifolds in $\mathbb{R}^{n+1}$, Comm. Anal. Geom. 9 (2001), 641-656.

[26] G. Perelman, The entropy formula for the Ricci flow and its geometric applications, arXiv:math.DG/0211159.

[27] S. Pigola, M. Rigoli and A. G. SetTi, Some characterizations of space-forms, Trans. Amer. Math. Soc. 359 (2007), 1817-1828.

[28] R. Schoen, Analytic aspects of the harmonic map problem, In: "Seminar on Nonlinear Partial Differential Equations", Math. Sci. Res. Ins. Publ. 2, Springer, New York, 1984, 321-358.

[29] Z-M. SHEN, Some rigidity phenomena for Einstein metrics, Proc. Amer. Math. Soc. 108 (1990), 981-987.

[30] Z-M. SHEN, Rigidity theorems for nonpositive Einstein metrics, Proc. Amer. Math. Soc. 116 (1992), 1107-1114.

[31] W. SHI, Deforming the metric on a complete Riemannian manifold, J. Differential Geom. 30 (1989), 223-301.

[32] M.A. SINGER, Positive Einstein Metrics with small $L^{n / 2}$-norm of the Weyl tensor, Differential Geom. Appl. 2 (1992), 269-274.

[33] J. STREETS, The gradient flow of the $L^{2}$ curvature energy near the round sphere, Adv. Math. 231 (2012), 328-356.

[34] G. TIAN, Kähler-Einstein metrics on algebraic manifolds, Proc. of ICM 1990, Math. Soc. Japan (1991), 587-598.

[35] G. TIAN and J.VIACLOVSKY, Bach-flat asymptotically locally Euclidean metrics, Invent. Math. 160 (2005), 357-415.

[36] G. TIAN and J. ViaClOVSKY, Moduli spaces of critical Riemannian metrics in dimension four, Adv. Math. 196 (2005), 346-372.

[37] G. TIAN and J. VIACLOVSKY, Volume growth, curvature decay, and critical metrics, Comment. Math. Helv. 83 (2008), 889-911.

[38] H-W. XU and E-T. ZHAO, $L^{p}$ Ricci curvature pinching theorems for conformally flat Riemannian manifolds, Pacific J. Math. 245 (2010), 381-396.

Laboratoire de Mathématiques Jean Leray (UMR 6629)

Université de Nantes

2, rue de la Houssinière, B.P. 92208

44322 Nantes Cedex 3, France

Gilles.Carron@math.univ-nantes.fr 